\title{
Investigating Taste-liking with a Humanoid Robot Facilitator
}

\author{
Zhuoni $\mathrm{Jie}^{1}$ and Hatice Gunes ${ }^{2}$
}

\begin{abstract}
Tasting is an essential activity in our daily lives. Implementing social robots in the food and drink service industry requires the social robots to be able to understand customers' nonverbal behaviours, including taste-liking. Little is known about whether people alter their behavioural responses related to taste-liking when interacting with a humanoid social robot. We conducted the first beverage tasting study where the facilitator is a human versus a humanoid social robot with priming versus non-priming instruction styles. We found that the facilitator type and facilitation style had no significant influence on cognitive taste-liking. However, in robot facilitator scenarios, people were more willing to follow the instruction and felt more comfortable when facilitated with priming. Our study provides new empirical findings and design implications for using humanoid social robots in the hospitality industry.
\end{abstract}

\section{INTRODUCTION}

Food is one of the fundamental necessities in life. Estimating people's liking of the taste of food products is important for predicting customer experience and acceptance [1] in service industries. The majority of the methods for measuring taste-liking have focused on explicit methods based on self-reported ratings (e.g., [2]). These have limitations caused by time and ability to give feedback [1], difficulty in quantifying rating metrics [3], and inherent bias due to people's conscious and rational processing in answering the questions [4]. Automatic analysis of nonverbal behaviour can potentially be utilised to mitigate this issue.

Integrating social robots into service industries requires social robots to be able to analyse and understand human nonverbal behaviour. One such industry is the hospitality industry that includes activities such as food and drink service among others. Many customers may be curious to try different dishes (e.g., a spicy dish) and drinks (e.g., sweet wine) but need to be assured by the waiter that they will like it and it will not ruin their expectations and experience. One option is to invite the customer to taste a small portion of the drink or the spicy sauce and give them a reference for the actual dish or drink based on that. In such situations, a social robot employed in the role of a waiter or a facilitator needs to be able to analyse and understand the nonverbal behavioural aspects of the customer taste-liking and response, including their facial and bodily expressions and emotional aspects of their speech. Recent advances in the affective computing field enable the detection and the prediction of affective states from facial expressions [5], body behaviour [6], speech [7], and physiological measures [8], that can be integrated into social robotic waiters.

This work is supported by EPSRC under grant ref. EP/R030782/1.

${ }^{1} \mathrm{Z}$. Jie finalised this study at the University of Cambridge. She is with the College of Information Studies, University of Maryland, College Park, MD 20742, USA z jielumd. edu

${ }^{2} \mathrm{H}$. Gunes is with the Department of Computer Science and Technology, University of Cambridge, Cambridge, CB3 OFD, UK Hatice.Gunes@cl.cam.ac.uk
Measuring appreciation of food in an objective way using taste-induced facial expressions is challenging. In tasting, facial expressions are a spontaneous response to flavour, not directly indicative of people's inner emotional states [1]. As studied in [9], adults' facial expression responses may be more complicated than innate spontaneous taste responses to flavour stimuli. Unlike newborns, adults tend to smile in response to some unpleasant tastes serving communicative functions. Another challenge is the fact that there are no publicly available datasets of facial expressions of tasteliking. Dibeklioğlu and Gevers created a database asking 502 respondents to evaluate six beers [1], but the dataset is not publicly available for research purposes.

In this study, we investigate (1) whether and how people alter their taste-liking behaviour and facial responses when encountering various tastes, and (2) whether these behaviours differ when the facilitator for tasting is a human or a humanoid social robot. To the best of our knowledge, this is the first study to investigate how a robot facilitator can influence people's taste-liking and interaction experience. Detailed analyses and understanding of people's taste-liking behaviour provided by this study may lead to insights that inform future robot designs with automatic taste-liking estimation capabilities.

\section{RELATED WORK}

\section{A. Facial Reactions to Tastes}

Facial reactions have been regarded as a powerful source of information to study taste-elicited affect in humans. Studies have consistently demonstrated newborns showing expressions indicating pleasure in response to sweet flavours, and indicating negative emotions in response to sour, bitter, and sometimes salty flavours [9]. People's innate behaviour to show a preference for sweetness and aversion to bitterness is found to be more pronounced in childhood than in adulthood, and is independent of culture [10]. People's appreciation and dislike of different flavours can cause corresponding facial reactions. According to Horio, adults show greater facial responses to disliked tastes than to liked tastes [11]. Negative reactions are found to occur more quickly than positive ones in adults and children [12]. There have been studies investigating the specific facial reactions elicited by tastes. For instance, the bitter taste is found to cause consistent facial responses of nose wrinkling, furrowing of the forehead, as well as mouth opening and brow lowering after swallowing [13].

\section{B. Automatic Facial Reaction Analysis}

Facial gestures and movements are mostly analysed in terms of the emotional information they communicate which has led to the development of automatic facial expression recognition (FER) systems and tools. The research field of 
FER has seen significant progress in recent years due to the availability of novel sensors, publicly available datasets, crowdsourced labels, and novel machine learning techniques. FER approaches usually extract hierarchical feature representations using carefully hand-crafted features [14] or, more recently, data-driven methodologies [15], to analyse and understand human facial expressions. The recent success of deep learning has further enhanced their performance by reducing the dependency on the choice of features used and learning these directly from data [16].

The Facial Action Coding System (FACS) [17] is another way of (manually) quantifying and detecting subtle changes in facial features, with a catalogue of 44 unique action units (AUs) that correspond to the movements of the face's individual 27 muscles. Previous works have shown that facial AUs can communicate positive and negative emotional tones [18] and various automatic systems have been developed to analyse facial AUs (see [19] for a survey).

The study presented in this paper uses the off-the-shelf open toolkit OpenFace [20] for automatic detection of facial AU presence and intensity, and for automatically analysing facial reactions to different tastes.

\section{Humanoid Social Robots for Service Industries}

Robotic technologies have already started influencing service industries. The first hotel predominantly staffed by robots, Henn-na, features robotic staff including front desk agents, porters, in-room assistants, and cleaners [21]. In 2014, a delivery robot developed by Savioke was tested in Aloft hotels [22]. Humanoid social robot Pepper has been employed by Pizza Hut to take customer orders and accept payments [23]. Chung et al. conducted four exploratory studies of the potential adoption of social robots for collecting customer feedback in hospitality industry [24]. They suggested hotel robots gather customer feedback in the context of the service and keep their interactions with guests brief, while keeping privacy concerns for customers and letting hotel staff incorporate their domain expertise into the robots' user interfaces.

Service industry robotic technologies' social influences have also been studied. Lee et al. designed a social robot snack delivery service for the workplace [25]. Over a fourmonth field study, they found employees extended the social roles of the robot beyond a delivery person, attaching several different roles to it. The robot created a ripple effect in the workplace, triggering new behaviours in employees. Herse $e t$ al. investigated robot persuasion for food recommendation and found that human-like features of an agent may contribute to boosting persuasion in recommendation systems [26]. However, none of these works studied whether and how a robot facilitator can influence people's taste-liking and interaction experience.

\section{BEVERAGE TASTING STUDY}

This study focused on people's cognitive liking (selfreported liking), which constitutes the basis for a future study on automatic taste estimation. We designed a beverage tasting study to investigate the following research questions: (1) whether and how people alter their taste-liking behaviour and facial responses when encountering various tastes, and

\begin{tabular}{ccc}
\hline No. & Session 1 & Session 2 \\
\hline 1 & Human * Non-priming * 5 flavours & Robot * Priming * 5 flavours \\
2 & Robot * Priming * 5 flavours & Human * Non-priming * 5 flavours \\
3 & Robot * Non-priming * 5 flavours & Human * Priming * 5 flavours \\
4 & Human * Priming * 5 flavours & Robot * Non-priming * 5 flavours \\
\hline
\end{tabular}

TABLE I: A list of study conditions for each participant.

(2) whether these behaviours differ when the facilitator is a human or a humanoid social robot with priming versus non-priming instruction styles. We situated the experiment in the Usability Laboratory of the Department of Computer Science and Technology, University of Cambridge. The study was approved by the Ethics Committee of the department.

\section{A. Study Design}

We considered three independent variables: flavour (i.e. sour, sweet, bitter, spicy, and salty), facilitator (i.e. either a human or a humanoid social robot), and instruction style (i.e. either with priming or non-priming), thus leading to a $5 \times 2^{2-1}$ fractional factorial design. Each participant attended two experiment sessions. For each of the two sessions, participants evaluated five beverages interacting with a human/robot under priming/non-priming instructions (facilitators and facilitation styles were consistent within each session), leading to ten tastings in total for each participant. As shown in Table 1, each participant attended one of the four conditions across two sessions. The order was randomised.

Facilitator conditions. During the human facilitator condition, the participants interacted with a human facilitator by responding to instructions to follow the task flow. During the robot facilitator condition, the experiments were performed in the same structured routine but led by a humanoid robot. Two warm-up questions were used to familiarise participants with the conversation style.

Priming and non-priming instruction conditions. In the priming condition, the flavour information was communicated by the facilitator saying e.g.: "Please pick up your cup and take a sip from this sour drink.”, while in the nonpriming condition the facilitator said: "Please pick up your cup and take a sip from this drink."

Participants. Twenty-seven volunteers (9 males and 18 females; mean age $=22.25$ years, $\mathrm{SD}=3.58$ years) were recruited from the University of Cambridge community via social media, e-mail, and website.

Materials. The five beverages used in the study were grape juice, lemonade, dark roast coffee, salty water, and nonalcoholic ginger beer with pepper sauce. We chose liquids to minimise the possible facial movements caused by chewing that might interfere with facial reaction analysis. The stimuli were presented in disposable paper cups. Straws were used to control the amount of each sip. The cup openings were covered so that participants could not guess visually or by odour the content of the cups before tasting the stimuli. Samples were served one at a time, $50 \mathrm{ml}$ per product. Participants did not have to consume all of the sample. In each session, the six cups (including a cup of water and five beverages) were placed on a table in front of the participant.

Robot Platform. The humanoid robot used in this study is Pepper (version 1.7 and body type V16) developed by 


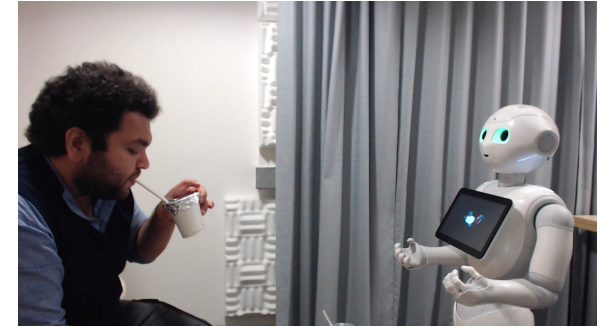

Fig. 1: Setup of the beverage tasting study.

SoftBank Robotics [27]. This robot is commercially available and has been widely used in Human-Robot Interaction (HRI) studies e.g., [28]. In this study, the Pepper robot was programmed to communicate and engage with participants through gestures, dialogic speeches, and LED lights in a Wizard-of-Oz manner (partially operated by the experimenter).

Procedure. All participants were asked to abstain from eating or drinking for two hours before arrival at the lab. After giving informed consent, participants filled in pre-study questionnaires. To familiarise participants with the tasting procedure and the use of the rating scales, a test trial was conducted with $50 \mathrm{ml}$ of water. Participants were told in advance not to swallow the drink right away, put down the cup, look forward and let the sip stay for approximately three seconds. There was a period of approximately 60 s between the presentation of one stimulus and the next, including the time to complete a post-study evaluation questionnaire. After each tasting sample, participants completed the questionnaire immediately, leading to 10 evaluations for each participant.

\section{B. Data Collection}

1) Questionnaire Data: The participants were asked to complete 11 questionnaires separately, one prior to the interaction session, and the other ten after each tasting instance.

The pre-study questionnaire aimed to record demographics as well as self-reported hunger and thirstiness before the study, and assess Big Five personality traits [29]. In this study, we used the Mini-IPIP [30]. The post-study questionnaires consisted of three sections on a 7-point Likert scale: taste-liking evaluation, taste perception scale, and interaction experience evaluation. Participants reported their taste-liking as well as perceptions after each taste and rated their interaction experience at the end of each session. At the end of the second session, participants' initial liking scores about the four ingredients involved in the beverages, namely grape, lemon, coffee, and ginger, were also self-reported. The first section consisted of eight questions adapted from measurement variables in [31] assessing overall liking score $(\mathrm{OL})$, food quality $(\mathrm{FQ})$, customer satisfaction (CS), and behavioural intentions (BI). In the second section, we recorded participants' perceptions regarding the five basic tastes. The third section contained three questions which evaluated the participants' degree of comfort interacting with the facilitator, and measured their impressions about adapting to and following the instructions.

2) Video Data: Nonverbal reactions (face, upper body and audio) during the entire session were captured using a
Logitech C920 high definition webcam positioned frontally to capture upper body and face (see Fig. 11). Videos were recorded with a resolution of $1280 \times 720$ pixels at a rate of 23 frames per second under controlled illumination conditions. Each subject had a recording of about 25 minutes. For further analysis, we segmented each recording into short clips. Each clip contains a tasting sample. For some tasting samples, participants had more than one tasting attempt, resulting in several clips of one tasting sample. This yielded in 377 clips in a total of about $107 \mathrm{~K}$ frames, with 197 clips of human facilitator, 180 clips of robot facilitator, 141 clips of priming facilitation style, and 236 clips of non-priming facilitation style.

3) Semi-structured Interview: The semi-structured interview schedule consisted of the following themes: reflections and comments on the humanoid social robot interaction experience; opinions about the instruction styles and their possible effects on anticipations, liking scores, and behaviours; and other comments, questions, and suggestions.

\section{Data Analysis}

1) Analysis of Taste-Liking: First, we examined the reliability of multi-item scales for each construct by measuring internal consistency. Internal consistency evaluates the quality of taste-liking measurements based on correlations between different questionnaire items that contribute to measuring the same consumer liking trait. We use standardised Cronbach's $\alpha$ to measure internal consistency of customer satisfaction $(\alpha=0.97)$ and behaviour intention $(\alpha=0.95)$.

An omnibus test was employed as the first step to detect whether the five beverages and four interaction conditions had caused a significant overall liking difference. Due to the ordinal nature of the Likert-scaled data, ordinal logistic regression (OLR) was implemented. The participants had significant $(p=0.0001)$ liking difference among the five flavours of the beverages, which demonstrated the five beverages did cause liking differences in participants. The estimated OLR coefficients for facilitator $(p=0.90)$ and facilitation style $(p=0.91)$ were not significant. We further tested whether or not the facilitators and facilitation styles had caused a taste-liking difference using two Mann-Whitney $U$ tests. No significant differences were found in these tests, demonstrating that facilitators and instruction styles did not cause a significant difference in taste-liking scores.

2) Analysis of Facial Reactions: In order to conduct facial reaction analysis to various tastes, we first created groundtruth taste-liking labels. Similar to [1], we mapped the overall liking scores from 7-point scale to 3-point scale (1 and 2 indicating dislike; 3, 4, and 5 indicating neutral state; 6 and 7 indicating liking), and they were used as class labels. The resulting database is composed of 172 dislike, 140 neutral, and 65 liking video clips.

To conduct facial reaction analysis, we focused on $\mathrm{AU}$ features as the information source providing affective cues for self-reported taste-liking. Using the existing facial behaviour analysis toolkit OpenFace [20], AU intensity and presence were automatically coded. Full names of the AU labels detected in this study and the total number of frames and videos in which each $\mathrm{AU}$ was present are recorded in Table 2. To study whether certain AUs are more intense 


\begin{tabular}{llcc}
\hline AU & Full name & Frames present & Videos present \\
\hline AU1 & Inner Brow Raiser & 17410 & 340 \\
AU2 & Outer Brow Raiser & 21180 & 359 \\
AU4 & Brow Lowerer & 25374 & 239 \\
AU5 & Upper Lid Raiser & 39339 & 319 \\
AU6 & Cheek Raiser & 16231 & 209 \\
AU7 & Lid Tightener & 27050 & 286 \\
AU9 & Nose Wrinkler & 8801 & 275 \\
AU10 & Upper Lip Raiser & 20939 & 262 \\
AU12 & Lip Corner Puller & 26193 & 311 \\
AU14 & Dimpler & 61854 & 367 \\
AU15 & Lip Corner Depressor & 23889 & 372 \\
AU17 & Chin Raiser & 40211 & 377 \\
AU20 & Lip Stretched & 15735 & 354 \\
AU23 & Lip Tightener & 43072 & 331 \\
AU25 & Lips Part & 21703 & 377 \\
AU26 & Jaw Drop & 25741 & 376 \\
AU45 & Blink & 28613 & 372 \\
\hline
\end{tabular}

TABLE II: AU label names and the total number of frames and videos in which each AU was present.

and appear more frequently for disliked flavour stimuli than liked flavour stimuli, one-tailed Mann-Whitney $U$ tests were conducted. For each AU, their presence ratios and average intensities in videos were compared grouping by disliked and liked video labels. The null hypotheses are: 1) AUn's (n denotes all the AU label numbers) presence ratios are higher in disliked videos than in liked videos; and 2) AUn's average intensities are higher in disliked videos than in liked videos. AU1, AU2, AU4, AU9, AU10, AU12, AU14, and AU23 showed a significant difference in presence ratios, demonstrating more frequent appearances in disliked videos. AU1, AU2, AU4, AU6, AU17, AU20, AU23, AU25, and AU26 showed a significant difference in average intensities, demonstrating higher intensities in disliked videos. These differences suggest that AU frequency and intensity features can be used for automatic prediction of taste-liking.

3) Analysis of Interaction Experience: To determine whether the facilitators and instruction styles had an association with interaction experience, OLR was adopted. The three questions measured participants' willingness to follow instructions (Q1), speed to adjust to the facilitation styles (Q2), and comfort in interactions (Q3). A Pearson correlation test was run to check if there was any correlation between these three question measurements and also to check the strength of the correlation. The Pearson correlations were estimated between each pair of the three components (Q1 \& Q2: $r=0.46, p<0.001$; Q2 \& Q3: $r=0.35, p=0.01$; Q1 \& Q3: $r=0.67, p<0.001)$. Based on these correlation scores, the questions measuring three aspects of interaction experience were analysed independently.

To eliminate potential learning and sequence effects and avoid potential confounding factors, we focused on analysing session one. Three OLR model fittings showed significant effects of controlling methods regarding facilitation styles on scores of the questions (Q1 $p_{\text {style }}=0.098, \mathrm{Q} 2 p_{\text {style }}=$ $0.047 *, \mathrm{Q} 3 p_{\text {style }}=0.026 *$ ), and no significant effect regarding facilitators $\left(\mathrm{Q} 1 p_{\text {facilitator }}=0.370, \mathrm{Q} 2 p_{\text {facilitator }}=\right.$ $0.662, \mathrm{Q} 3 p_{\text {facilitator }}=0.054$.). The result showed that facilitation styles can significantly affect interaction experience in the first session, while the facilitator had a limited influence on people's interaction experience.

We then investigated potential interaction effects between the facilitator and facilitation style conditions. According to the interaction plots shown in Fig. 2, for Q1 and Q3, the scores did not vary much for human facilitator condition during priming and non-priming trails, while for robot facilitator condition the scores in priming condition were constantly higher than in non-priming condition. This indicated that participants were more willing to be instructed in priming style when interacting with a robot, and felt more comfortable when explicitly instructed by a robot facilitator compared to a human facilitator. The speed to adjust to instructions (Q2) was consistently higher for priming condition compared to non-priming for both facilitators.

For post-hoc tests, three one-tailed independent 2-group Mann-Whitney $U$ tests were implemented to investigate which facilitator led to higher self-reported interaction experience scores. Ratings for Q3 were statistically significant ( $p=0.034)$, indicating that the participants tended to feel significantly more comfortable interacting with a human.

In human-robot sessions, three $U$ tests were used to investigate which facilitation style led to higher self-reported interaction experience scores. Ratings for Q1, Q3 were both statistically significant $\left(p_{1}=0.016, p_{3}=0.023\right)$ regarding nonpriming versus priming instructions, suggesting participants were more willing to follow priming instructions and felt more comfortable when instructed by a robot. The ratings for Q2 were not statistically significant. These results show that, in terms of participants' willingness to follow instructions and feeling of comfort regarding facilitation styles, the nonpriming facilitation style had a significant negative influence on participants' experience in robot facilitator condition. In contrast, $U$ tests were also conducted for human facilitator sessions, with no statistically significant difference found regarding facilitation styles.

4) Semi-structured Interview Analysis: Semi-structured interview data were coded to generate key qualitative results.

Robot behaviour. Due to the nature of the study, the Pepper robot was programmed to verbally respond to a limited number of words/sentences so that the experiment flow was under control. After initial adaptation, some participants found this instruction style easy to follow, as seen in P2's comments in Table 3. If the participant was not ready, the experiment would not move onto the next stage. Letting users decide whether to continue or not resulted in the perception of more sense of control. P3 reported enjoying the predictability of the robot's instructions. On the other hand, P4's comments oppose this view, and are in line with the theory of [32] that the perceived repetitiveness and hence predictability of a robot can lead to diminished perception of the robot's intelligence by the user, and ultimately to a loss of user trust in this robot facilitator. This might prevent participants from naturally interacting with the facilitator and expressing their true feelings. Therefore, careful consideration is needed for adding a suitable degree of variety to the robot's utterances.

Following instructions. Sometimes, participants were not willing to follow the instructions of the Pepper robot. As shown in Table 3, P5 regarded responding to a human as 


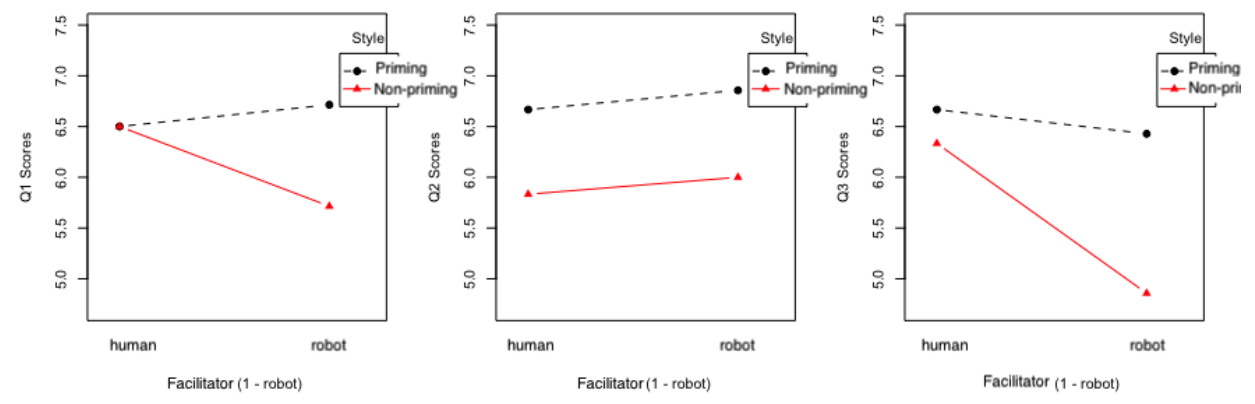

Fig. 2: Interaction plots showing interaction effects between the facilitator and facilitation style conditions on scores of Q1 (left), Q2 (middle), and Q3 (right).

more meaningful and rewarding, and felt that it was unnatural to interact with the robot. Some participants were even afraid of interacting with Pepper. P10 was confused with the experimental procedures at the beginning. She chose not to ask Pepper questions because she did not expect any answers. P21 had a low expectation of the robot's intelligence and social (support) abilities. These indicate that when designing robot facilitators, how to appropriately communicate and demonstrate the robot's intelligence and (social) capabilities need to be addressed.

Robot likeability. 7 out of 27 participants explicitly mentioned that Pepper was (visually) cute due to its appearance, LED colours, and body gestures. Whether they viewed the robot as cute or not did not change their opinions about the interaction experience itself. However, interacting with a robot facilitator that is perceived as cute may serve as a social function, as mentioned by P6: "The robot was cute, and interacting with it made me happier, especially when I tasted some awful drinks."

Facilitation style. The priming facilitation style seemed to provide participants with information that helped them formulate expectations before the tasting took place. This might have influenced their behaviours as commented by $\mathrm{P} 4$. At times the information provided prior to tasting could elicit emotions in participants even before they tasted the different flavours. According to P12, these pre-elicited emotions may impact taste-liking and self-reported evaluations. However, the influence of priming instructions seemed to vary from person to person, as seen in the comments of P14 and P20. Such psychological and behavioural factors may indeed influence the creation of robot facilitators that are capable of automatically estimating taste-liking.

\section{SUMMARY AND CONCLUSIONS}

We have presented the first beverage tasting study investigating people's behaviour and interaction experience when the facilitator for tasting is a human vs a humanoid social robot. Our study has shown that in a tasting context, people were more willing to follow the instructions when the facilitator is a robot with priming and they felt more comfortable when facilitated with priming by the robot. Facial AU analyses suggested that there are significant differences in occurrence frequency and intensity of certain AUs, which can be successfully utilised for training automatic taste-liking predictors. To this aim, other nonverbal cues such as body language and intonation can also be employed.

We further derive the following three design implications that should be taken into account when designing robot facilitators for the hospitality industry:

1) Design for controllability: Letting the users/customers decide whether to continue the interaction flow can result in the perception of more sense of control. A reasonable amount of controllability for customers (users) should be allowed to achieve this.

2) Design for predictability: People enjoy varying degrees of predictability. Adjusting and appropriately communicating robot facilitator's intelligence and (social) capabilities through its response behaviours, while maintaining reasonable control of the interaction flow, can make the interaction feel more natural.

3) Design for likability: The appearance features and nonverbal behaviours of robot facilitators can play an important role in building social relationships with the customers, which in turn may help with eliciting positive customer emotions and reactions.

It is our hope that the findings and the design implications offered by this work will assist the development of effective, efficient and engaging robot facilitators for the hospitality industries.

\section{ACKNOWLEDGMENTS}

We would like to thank Indu Bodala and Nikhil Churamani from the AFAR Lab at the Department of Computer Science and Technology, Cambridge, Wenxuan Mou from Queen Mary University of London, and Alan Blackwell, Simone Schnall, Paul Fletcher and Elena Mainetto from the University of Cambridge for their valuable comments, insights and suggestions.

\section{REFERENCES}

[1] H. Dibeklioglu and T. Gevers, "Automatic estimation of taste liking through facial expression dynamics," IEEE Transactions on Affective Computing, vol. 11, pp. 151-163, 2020.

[2] S. C. King, H. L. Meiselman, and B. T. Carr, "Measuring emotions associated with foods: Important elements of questionnaire and test design," Food Quality and Preference, vol. 28, no. 1, pp. 8-16, 2013. 


\begin{tabular}{|c|c|}
\hline Participant & Quotes about repetitive and predictable robot behaviour \\
\hline P2 & $\begin{array}{l}\text { The interaction process was smooth, although it would be better if the robot could speak slower and pause } \\
\text { more. It's nice to have users to control the experiment flow, so that we can have some rest if we want. }\end{array}$ \\
\hline P3 & It (the interaction) was not complicated and only had one thread. I could easily follow the facilitation. \\
\hline P4 & $\begin{array}{l}\text { The interaction process was good, but not so flexible. I was still more willing to express my opinions when } \\
\text { interacting with a human, and I stopped think-aloud when interacting with a robot. }\end{array}$ \\
\hline Participant & Quotes about obeying facilitator \\
\hline P5 & $\begin{array}{l}\text { The interaction was good, but I still preferred to speak to a human. I will actively respond to the human's } \\
\text { instructions, but not to the robot's. }\end{array}$ \\
\hline P10 & I still trust more in humans. I dared not communicate with and ask questions to Pepper. \\
\hline $\mathrm{P} 21$ & $\begin{array}{l}\text { I would not say extra words to the robot, because I knew I should only respond to its instructions to indicate } \\
\text { I was done. I would not ask it questions or check with it. }\end{array}$ \\
\hline Participant & Quotes about facilitation style \\
\hline P4 & $\begin{array}{l}\text { It did influence my behaviours. I was a bit afraid when faced with the unknown. If I were told what I was } \\
\text { going to taste, I would probably take a big sip, and if not, I would probably take a small sip. }\end{array}$ \\
\hline $\mathrm{P} 12$ & I felt sad after realising good-taste ones were done. \\
\hline P14 & $\begin{array}{l}\text { Some people may be disappointed or excited to have an expectation for the drinks. But to me, I think it did } \\
\text { not influence that much. }\end{array}$ \\
\hline $\mathrm{P} 20$ & $\begin{array}{l}\text { I became more prepared when told about the flavour type, but this did not affect any of my evaluations or } \\
\text { perceptions. }\end{array}$ \\
\hline
\end{tabular}

TABLE III: Selected comments from the interviews.

[3] D. McDuff, R. El Kaliouby, et al., "Automatic measurement of ad preferences from facial responses gathered over the internet," Image and Vision Computing, vol. 32, no. 10, pp. 630-640, 2014.

[4] E. P. Köster, "The psychology of food choice: some often encountered fallacies," Food Quality and Preference, vol. 14, no. 5-6, pp. 359-373, 2003.

[5] L. Danner, L. Sidorkina, et al., "Make a face! implicit and explicit measurement of facial expressions elicited by orange juices using face reading technology," Food Quality and Preference, vol. 32, pp. 167$172,2014$.

[6] A. Kleinsmith and N. Bianchi-Berthouze, "Affective body expression perception and recognition: A survey," IEEE Transactions on Affective Computing, vol. 4, no. 1, pp. 15-33, 2012.

[7] B. Schuller, G. Rigoll, and M. Lang, "Speech emotion recognition combining acoustic features and linguistic information in a hybrid support vector machine-belief network architecture," in 2004 IEEE International Conf. on Acoustics, Speech, and Signal Processing, vol. 1. IEEE, 2004, pp. I-577.

[8] A. Bechara, H. Damasio, et al., "The iowa gambling task and the somatic marker hypothesis: some questions and answers," Trends in cognitive sciences, vol. 9, no. 4, pp. 159-162, 2005.

[9] R. Weiland, H. Ellgring, and M. Macht, "Gustofacial and olfactofacial responses in human adults," Chemical senses, vol. 35, no. 9, pp. 841$853,2010$.

[10] D. R. Reed, T. Tanaka, and A. H. McDaniel, "Diverse tastes: Genetics of sweet and bitter perception," Physiology \& behavior, vol. 88, no. 3, pp. 215-226, 2006.

[11] T. Horio, "Emg activities of facial and chewing muscles of human adults in response to taste stimuli," Perceptual and motor skills, vol. 97, no. 1, pp. 289-298, 2003.

[12] J. E. Steiner, "The gustofacial response: observation on normal and anencephalic newborn infants." 1973.

[13] J. E. Steiner, D. Glaser, et al., "Comparative expression of hedonic impact: affective reactions to taste by human infants and other primates," Neuroscience \& Biobehavioral Reviews, vol. 25, no. 1, pp. 53-74, 2001.

[14] L. Zhong, Q. Liu, et al., "Learning active facial patches for expression analysis," in IEEE Conf. on Computer Vision and Pattern Recognition, 2012, pp. 2562-2569.

[15] S. Li and W. Deng, "Deep facial expression recognition: A survey," CoRR, vol. abs/1804.08348, 2018.

[16] D. Kollias and S. Zafeiriou, "Training Deep Neural Networks with Different Datasets In-the-wild: The Emotion Recognition Paradigm," in Proc. IJCNN, 2018, pp. 1-8.

[17] E. Friesen and P. Ekman, "Facial action coding system: a technique for the measurement of facial movement," Palo Alto, vol. 3, 1978.

[18] P. D. Bolls, A. Lang, and R. F. Potter, "The effects of message valence and listener arousal on attention, memory, and facial muscular responses to radio advertisements," Communication Research, vol. 28, no. 5, pp. 627-651, 2001.
[19] B. Martinez, M. F. Valstar, et al., "Automatic analysis of facial actions: A survey," IEEE Tran. on Affective Computing, vol. 10, no. 3, pp. 325347, 2019.

[20] T. Baltrusaitis, A. Zadeh, et al., "Openface 2.0: Facial behavior analysis toolkit," in 2018 13th IEEE International Conf. on Automatic Face \& Gesture Recognition (FG 2018). IEEE, 2018, pp. 59-66.

[21] R. M. (2017) Inside japan's first robot-staffed hotel. the guardians. [Online]. Available: https://www.theguardian.com/travel/2015/aug/14/ japan-henn-na-hotel-staffed-by-robots

[22] M. J. (2014) 'beep' says the bellhop. the new york times. [Online]. Available: https://www.nytimes.com/2014/08/12/technology/ hotel-to-begin-testing-botlr-a-robotic-bellhop.html?_r=0

[23] C. S. (2016) Pizza hut hires robot waiters to take orders and process payments at its fastfood restaurants. mirror. [Online]. Available: https://www.mirror.co.uk/tech/pizza-hut-hires-robot-waiters-8045172

[24] M. J.-Y. Chung and M. Cakmak, “"how was your stay?": Exploring the use of robots for gathering customer feedback in the hospitality industry," in 2018 27th IEEE International Symposium on Robot and Human Interactive Communication (RO-MAN). IEEE, 2018, pp. $947-$ 954.

[25] M. K. Lee, S. Kiesler, et al., "Ripple effects of an embedded social agent: a field study of a social robot in the workplace," in Proc. of the SIGCHI Conf. on Human Factors in Computing Systems. ACM, 2012, pp. 695-704.

[26] S. Herse, J. Vitale, et al., "Bon appetit! robot persuasion for food recommendation," in Companion of the 2018 ACM/IEEE International Conf. on Human-Robot Interaction. ACM, 2018, pp. 125-126.

[27] S. Robotics, "Pepper," Softbank Robotics, 2016.

[28] M. Niemelä, P. Heikkilä, and H. Lammi, "A social service robot in a shopping mall: expectations of the management, retailers and consumers," in Proc. of the Companion of the 2017 ACM/IEEE International Conf. on Human-Robot Interaction. ACM, 2017, pp. 227-228.

[29] O. P. John, S. Srivastava, et al., "The big five trait taxonomy: History, measurement, and theoretical perspectives," Handbook of personality: Theory and research, vol. 2, no. 1999, pp. 102-138, 1999.

[30] M. B. Donnellan, F. L. Oswald, et al., "The mini-ipip scales: tiny-yeteffective measures of the big five factors of personality." Psychological assessment, vol. 18, no. 2, p. 192, 2006

[31] K. Ryu, H.-R. Lee, and W. Gon Kim, "The influence of the quality of the physical environment, food, and service on restaurant image, customer perceived value, customer satisfaction, and behavioral intentions," International journal of contemporary hospitality management, vol. 24, no. 2, pp. 200-223, 2012.

[32] J. Fasola and M. J. Mataric, "Robot exercise instructor: A socially assistive robot system to monitor and encourage physical exercise for the elderly," in 19th International Symposium in Robot and Human Interactive Communication. IEEE, 2010, pp. 416-421. 